\title{
ASSESSMENT OF SCLEROCORNEAL INCISION ARCHITECTURE AFTER PHACOEMULSIFICATION USING AS-OCT
}

\author{
Jana Simova, Mladena Radeva, Dimitar Grupchev, Christina Grupcheva \\ Department of Ophthalmology and Visual Sciences, Faculty of Medicine, \\ Medical University of Varna
}

\begin{abstract}
INTRODUCTION: The construction of the main incision is crucial for the outcome of cataract surgery. There are many considerations including: wound preparation and stability, surgically induced astigmatism, stability of anterior chamber, and wound closure stability with rising intraocular pressure (IOP). Irrespective of modality, the main goal is to achieve stable watertight self-sealing wound in order to avoid possible intra- and postoperative complications. Therefore, evaluating the architecture and healing of the surgical wound would be of benefit, as the integrity of incisions may decrease the risk of endophthalmitis or other problems. Anterior segment OCT (AS-OCT) provides the opportunity to visualize anterior chamber structures and cornea in particular. In the interest of the current study this was used to examine the anatomy of the main incision in vivo and evaluate its changes over time.
\end{abstract}

AIM: The aim of this article is to examine the architecture of the main incision in vivo and evaluate its changes over time, using anterior segment optical coherence tomography (AS-OCT).

MATERIALS AND METHODS: Patients diagnosed with senile cataract, scheduled for surgery, were enrolled in this prospective study. All underwent uneventful cataract surgery with phacoemulsification and intraocular lens (IOL) implantation, performed by the same experienced surgeon. A standardized technique of cataract extraction, under local anesthesia, was used. Patients with previous ocular surgery or trauma, and other ocular pathology, as well as cases with complications were not included in the study. Wound anatomy was visualized using AS-OCT (Topcon 3D SD OCT 2000) at three postoperative visits: day 1, day 7, and day 30. The following features were examined and analyzed: external gaping, endothelial (internal) alignment and gaping, localized DMD, loss of coaptation, wound retraction, and other changes on the external or the internal side of the wound.

RESULTS: Sixty eyes of 51 patients were examined, of whom 23 were men and 28 women, aged between 53 and 87 years (mean 72 years). The scleral tunnel incisions had a three-plane architecture and all of them were watertight with no leakage at any time. Several architectural features were assessed, including epithelial gaping, endothelial gaping and misalignment, local Descemet's membrane detachment (DMD).

CONCLUSION: AS-OCT is a risk-free, quick, high-informative method for visualization of the morphological characteristics of cataract surgical incisions and their evolution over time. Hence, this technolo-

Address for correspondence:

Jana Simova

Faculty of Medicine

Medical University of Varna

55 Marin Drinov

9002 Varna

e-mail: janasimova@abv.bg

Received: November 19, 2018

Accepted: December 28, 2018 gy is indispensable in evaluating the integrity of the wound, and the information obtained could be used to develop a consistent technique to create self-sealing incisions and decrease the risk of endophthalmitis.

Keywords: AS-OCT, cataract surgery, phacoemulsification, incision, cornea 
Jana Simova, Mladena Radeva, Dimitar Grupchev et al.

\section{INTRODUCTION}

The construction of the main incision is crucial for the outcome of cataract surgery. There are different techniques, with different advantages and disadvantages. There are many considerations including: wound preparation and stability, surgically induced astigmatism, stability of anterior chamber, and wound closure stability with rising intraocular pressure (IOP). Irrespective of modality, the main goal is to achieve stable watertight self-sealing wound in order to avoid possible intra- and postoperative complications $(1,2)$. Therefore, evaluating the architecture and healing of the surgical wound would be of benefit, as the integrity of incisions may decrease the risk of endophthalmitis or other problems. There are several options for assessment of the surgical incision: slit-lamp microscopy, confocal microscopy (3), ultrasound biomicroscopy (UBM) $(4,5)$, and optical coherence tomography (OCT) $(6,7)$. Fourier-domain OCT (FD-OCT) is a non-contact, noninvasive high-resolution technology enabling crosssectional imaging of ocular tissues. Anterior segment OCT (AS-OCT) provides the opportunity to visualize anterior chamber structures and cornea in particular (8-11)high resolution cross-sectional images of ocular tissues. Development of anterior segment OCT (AS-OCT. In the interest of the current study this was used to examine the anatomy of the main incision in vivo and evaluate its changes over time. Several architectural features were assessed, including epithelial gaping, endothelial gaping and misalignment, local Descemet's membrane detachment (DMD).

\section{MATERIALS AND METHODS}

The present study was conducted at the Department of Ophthalmology and Visual Sciences at Medical University of Varna and the Specialized Eye Hospital in Varna.

Patients diagnosed with senile cataract, scheduled for surgery, were enrolled in this prospective study. All underwent uneventful cataract surgery with phacoemulsification and intraocular lens (IOL) implantation, performed by the same experienced surgeon. A standardized technique of cataract extraction, under local anesthesia, was used. All incisions were performed as sclerocorneal tunnel (sclerocorneal incision, SCI), utilizing three different instruments for the three distinctive steps. Patients with previous ocular surgery or trauma, and other ocular pathology, as well as cases with complications were not included in the study.

\section{Cataract surgery}

After a fornix-based conjunctival peritomy, the main 3-plane incision was performed superiorly. The incision was centered on the steepest meridian (usually the vertical) and was done in three distinctive steps: (1) vertical semi-scleral incision $2 \mathrm{~mm}$ behind the limbus with a 15-degree blade; (2) scleral tunnel to the clear cornea with a crescent knife (approximately $3.5 \mathrm{~mm}$ long); (3) anterior chamber (AC) entering with a 2.2 keratome. Then a sideport incision was created at $20^{\prime}$ clock position (along the $60^{\circ}$ meridian) with a 15-degree blade. Viscoelastic was injected through the SCI to maintain AC and a curvilinear capsulorrhexis, followed by hydrodissection, were performed. Phacoemulsification was performed. Then, using a disposable injector, a foldable IOL was implanted into the capsular bag. The residual viscoelastic was removed by irrigation and/or aspiration and BSS was injected to restore the normal rigid state of the globe. The incisions were hydrated and tested for leakage. At the end of the surgery intracameral Cefuroxime was injected, subconjunctival antibiotic/steroid injection was given and a sterile eye patch was placed over the eye. Postoperative aftercare instructions were provided according to the normal protocol of the hospital. The therapeutic regimen included topical antibiotic and steroid (Tobramycin $3 \mathrm{mg} / \mathrm{mL}$-Dexamethasone $1 \mathrm{mg} / \mathrm{mL}$, Tobradex, Alcon; Levofloxacin $5 \mathrm{mg} / \mathrm{mL}$, Oftaquix coll., Santen) 5-7 times during the first 24 hours and then 5 times a day for one month.

Wound anatomy was visualized using AS-OCT (Topcon 3D SD OCT 2000) at three postoperative visits: day 1 , day 7 , and day 30 . The following features were examined and analyzed: external gaping, endothelial (internal) alignment and gaping, localized DMD, loss of coaptation, wound retraction and other changes on the external or the internal side of the wound.

\section{RESULTS}

Sixty eyes of 51 patients were examined, of whom 23 were men and 28 women, aged between 53 and 87 years (mean 72 years). 
The incisions had a three-plane architecture and all of them were watertight with no leakage at any time.

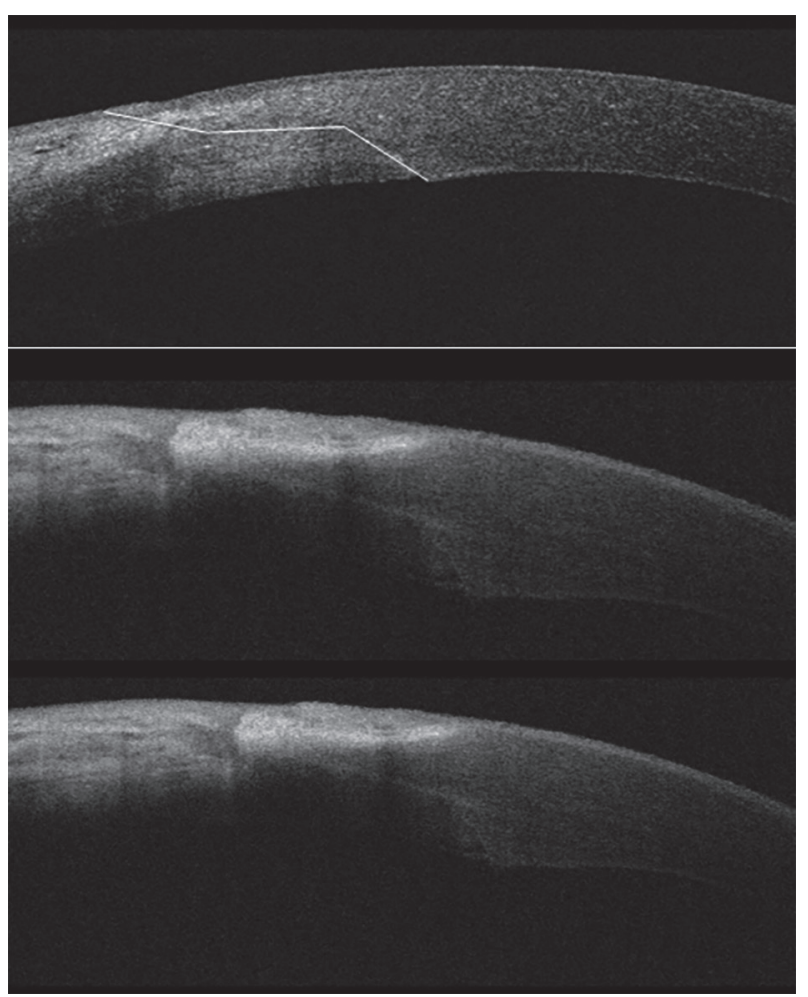

Fig. 1. Anterior segment optical coherence tomography (AS-OCT) images of cataract main incisions (3-planar),

$$
1^{\text {st }} \text { postoperative day. }
$$

\section{Changes on the Epithelial Side of the Wound}

Epithelial gaping was evident in 35 eyes and 21 eyes on the first and second postoperative visit, respectively, while on the last one such was not detected.

One or more epithelial bullae were evident in $3 \%$ of the cases on day 1 , and all of them were in conjunction with stromal edema and localized DMD. They resolved by the $7^{\text {th }}$ day after surgery.

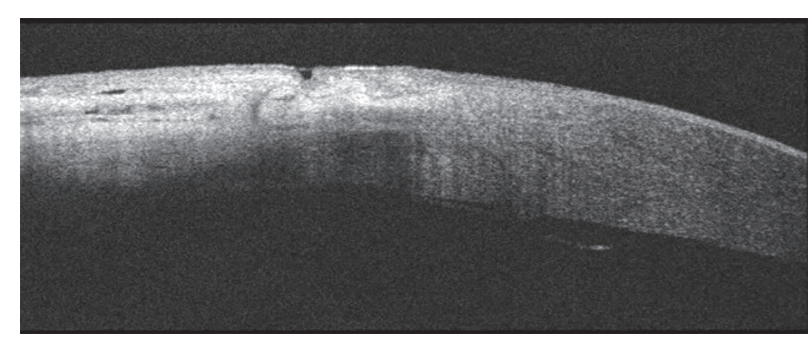

Fig. 2. AS-OCT image demonstrating epithelial gaping
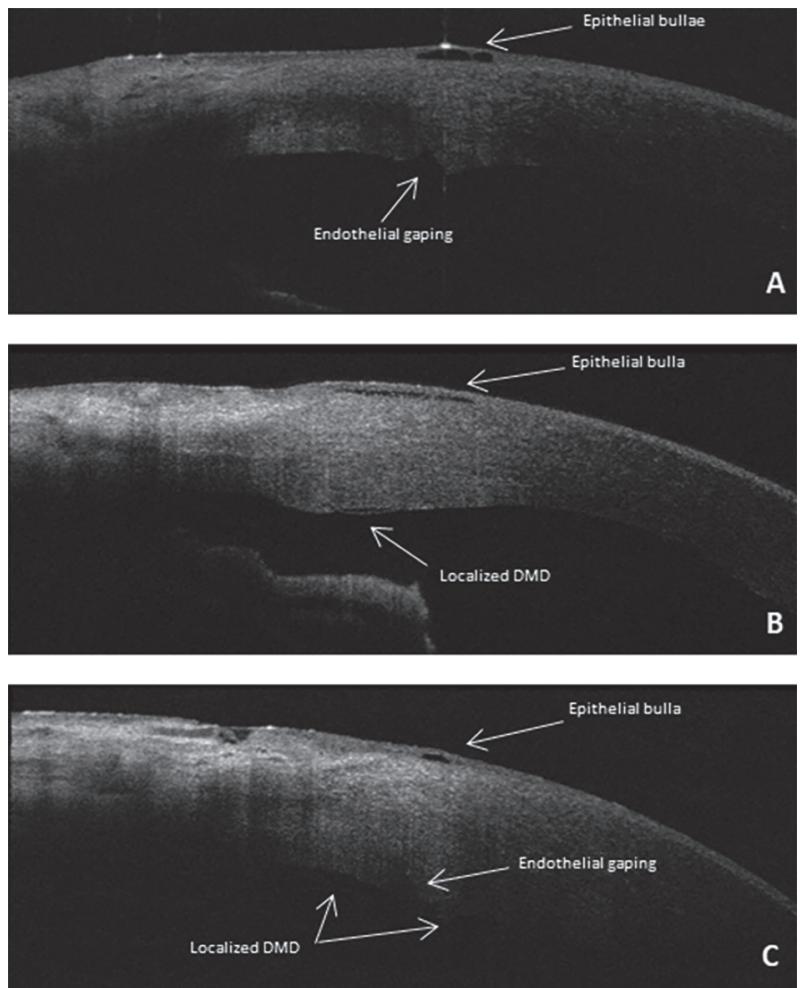

Fig. 3. AS-OCT images demonstrating epithelial edema and localized DMD (A, B), epithelial bullae, localized $D M D$, and endothelial gaping (C).

\section{Changes on the Endothelial Side of the Wound}

Endothelial gaping was observed in 34\%, 26\% and $8 \%$ of the cases on the first, seventh and thirtieth day after surgery, respectively; and the results for the endothelial misalignment were $24.5 \%, 18 \%$ and $9.8 \%$.
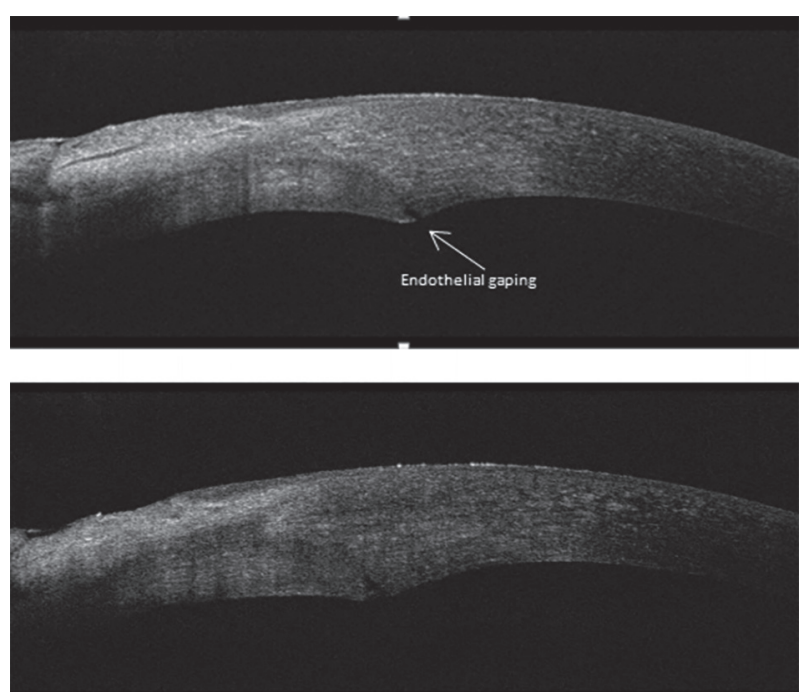

Fig. 4. AS-OCT images demonstrating endothelial gaping 
Jana Simova, Mladena Radeva, Dimitar Grupchev et al.

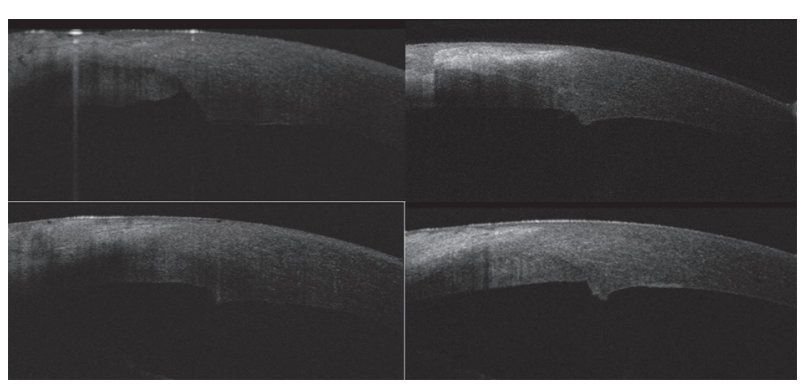

Fig. 5. AS-OCT images of endothelial misalignment.

Localized DMD was detected in $46 \%$ of the eyes on the first postoperative day, it decreased to $29.5 \%$ by the end of the first week, and on the last visit there was only one eye (1.7\%) with localized DMD.

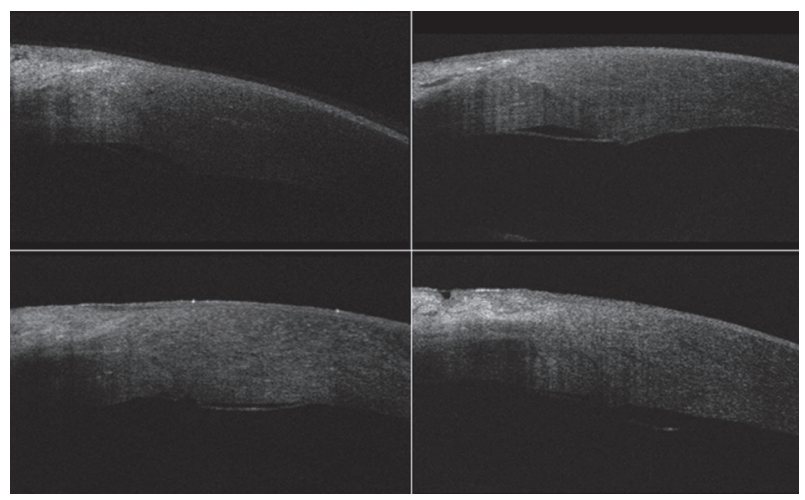

Fig. 6. AS-OCT images demonstrating localized DMD.

Posterior wound retraction was found in 8 eyes (13.3\%) but not before the third postoperative visit.

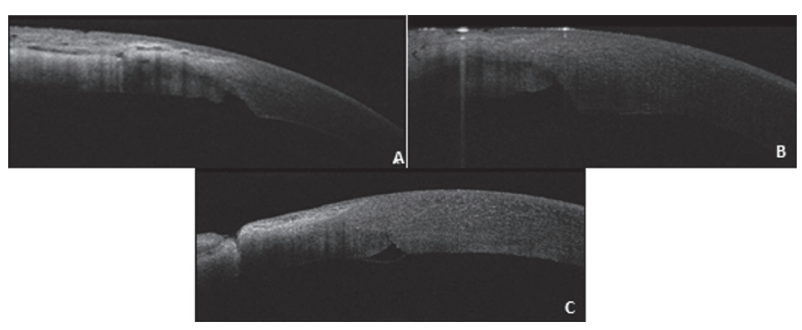

Fig. 7. AS-OCT images demonstrating wound retraction (A), or wound retraction in association with localized $\operatorname{DMD}(B, C)$

\section{DISCUSSION}

Several studies have demonstrated that incision integrity is mainly affected by two factors: mechanical injury and thermal injury. While the former is caused by surgical instruments, introduced into the wound, the latter is due to ultrasonic energy $(12-15)$. It has been found that the healing of the incision starts from the external part of the wound (16). As a result of the endothelial pump function, suction is generated within the wound, which opposes the wound margin (17). Epithelial gaping counteracts the suction and barrier mechanisms, and creates a risk of endophthalmitis (16). The incisions in our study were sclerocorneal, which is the reason our results could not be compared with those of the reviewed reports, for the reason they were evaluating clear corneal incisions (CCIs) (7,17-24).

Corneal endothelium plays a crucial role in maintaining corneal structure and transparency and could be damaged by several factors during phacoemulsification (25-27). Bullous keratopathy after cataract surgery is commonly due to endothelial dysfunction (28). In the current study epithelial bullae were evident on the external side of the wound but they could not be caused by wound leakage, as they were not covering its opening, but rather by localized endothelial dysfunction and stromal edema (18).

Endothelial gaping incidence varies widely throughout studies and our results were generally lower and consistent with some of them $(7,22,24)$. Some authors indicated endothelial gape could be explained with surgical instrument sizing or excessive ultrasound (US) power, corneal stromal edema and endothelial damage $(18,29)$. It has been suggested that internal gaping is dependent on IOP and mostly occurs in eyes with normal or low IOP $(30,31)$. All enrolled patients had normal IOP at every visit, although IOP readings are not included in this study.

Fine et al. (32) and Calladine and Tanner (33) noted that stromal hydration results in lower incidence of endothelial gape, which explained the difference of our results (as stromal hydration was performed routinely in all our cases). In comparison, Xia et al. (18), performed and had much higher incidence of gapping on the first postoperative day. Another study presenting higher incidence of internal gaping provided no data concerning stromal hydration (23). The evolution of surgical wound healing of our study confirmed other researchers' observations that endothelial gaping and misalignment improved over time by itself $(7,19,29,34)$. 
Localized DMD was found to be common after phacoemulsification, which was similar with other investigations $(18,20,33,35)$. Such a feature could be in correlation with stromal hydration, routinely performed by our experienced surgeon, and the subsequent mechanical forces exerted on the DM (33). Localized DMD might be caused by unintentional stripping during initial construction of the main incision or insertion of instruments during the procedure (35). Our study proved that localized DMD is not visible on slit-lamp in any of the cases $(18,36)$, thus confirming the advantages of AS-OCT in corneal imaging once again. Calladine et al. reported also that it could lead to extensive spontaneous peeling of DM (35), which fortunately, we did not encounter at any degree in any of our cases during the entire follow-up period.

Endothelial misalignment in the early postoperative period is more likely to occur with stromal hydration. However, the latter is applied to improve wound sealing and to prevent inflow of ocular surface fluid, thus countering the risk of endophthalmitis (33). This shows that stromal hydration, along with incisional size, is a crucial factor affecting the configuration and healing of clear corneal incisions. Our findings demonstrate contrasting incidence rates of internal misalignment as opposed to other studies $(7,22,23)$ and the difference might be a result of the difference in the incision type.

Two studies have reported the presence of posterior wound retraction. In one (20) the incidence was $33.3 \%$ at 2 to 3 weeks postoperatively with increasing percentages later on up to 3 years after cataract surgery, while the other (24) documented much lower rate of posterior wound retraction but both stated that it occurred no earlier than the second week after surgery. Our results differ from both reports probably due to the different, safer surgical technique.

Loss of coaptation is known to occur in the event of leaking wounds (33). It is reported it might influence wound structural integrity and increase the risk of endophthalmitis (19). We did not note any cases with loss of coaptation, which could be explained by the suggestion that it would be less likely to appear with a longer incision, because a lower proportion of the total length is misaligned (35). Moreover, the discrepancy between our results and other reports could be due to the fact our patients were examined 24 hours after surgery, while others' were evaluated in the immediate (30-minute) postoperative period (19) and 2 hours after surgery (24).

\section{CONCLUSION}

In conclusion, AS-OCT is a risk-free, quick, high-informative method for visualization of the morphological characteristics of cataract surgical incisions, and their evolution over time. Hence, this technology is indispensable in evaluating the integrity of the wound, and the information obtained could be used to develop a consistent technique to create self-sealing incisions and decrease the risk of endophthalmitis and other complications.

\section{REFERENCES}

1. Pham DT, Wollensak J, Liekfeld A. Self-closing corneoscleral tunnel incision in cataract surgery. Ophthalmologe. 1996;93(1):8-11.

2. Agrawal R. Wound construction in manual small incision cataract surgery. Indian J Ophthalmol. 2010;58(1):85. doi: 10.4103/0301-4738.58486.

3. Patel DV, Phang KL, Grupcheva CN, Best SJ, McGhee CNJ. Surgical detachment of Descemet's membrane and endothelium imaged over time by in vivo confocal microscopy. Clin Experiment Ophthalmol. 2004;32(5):539-42. doi: 10.1111/j.1442-9071.2004.00875.x.

4. Morinelli EN, Najac RD, Speaker MG, Tello C, Liebmann JM, Ritch R. Repair of Descemet's membrane detachment with the assistance of intraoperative ultrasound biomicroscopy. Am J Ophthalmol. 1996;121(6):718-20.

5. Pereira FAS, Cronemberger S. Ultrasound biomicroscopic study of anterior segment changes after phacoemulsification and foldable intraocular lens implantation. Ophthalmology. 2003 Sep;110(9):1799-806. doi: 10.1016/ S0161-6420(03)00623-7.

6. Schallhorn JM, Tang M, Li Y, Song JC, Huang D. Optical coherence tomography of clear corneal incisions for cataract surgery. J Cataract Refract Surg. 2008;34(9):1561-5. doi: 10.1016/j.jcrs.2008.05.026.

7. Torres LF, Saez-Espinola F, Colina JM, Retchkiman M, Patel MR, Agurto R, et al. In vivo architectural analysis of $3.2 \mathrm{~mm}$ clear corneal incisions for phacoemulsification using optical co- 
Jana Simova, Mladena Radeva, Dimitar Grupchev et al.

herence tomography. J Cataract Refract Surg. 2006;32(11):1820-6. doi: 10.1016/j.jcrs.2006.06.020

8. Jancevski M, Foster CS. Anterior segment optical coherence tomography. Semin Ophthalmol. 2010;25(5-6):317-23. doi: 10.3109/08820538.2010.518473.

9. Rio-Cristobal A, Martin R. Corneal assessment technologies: current status. Surv Ophthalmol. 2014;59(6):599-614. doi: 10.1016/j. survophthal.2014.05.001.

10. Bald M, Li Y, Huang D. Anterior chamber angle evaluation with Fourier-domain optical coherence tomography [Internet]. J Ophthalmol. 2012 [cited 2018 Aug 2]. Available from: https://www.hindawi. com/journals/joph/2012/103704/

11. Doors M, Berendschot TTJM, de Brabander J, Webers CAB, Nuijts RMMA. Value of optical coherence tomography for anterior segment surgery. J Cataract Refract Surg. 2010;36(7):1213-29. doi: 10.1016/j.jcrs.2010.05.002.

12. Bradley MJ, Olson RJ. A survey about phacoemulsification incision thermal contraction incidence and causal relationships. Am J Ophthalmol. 2006;141(1):222-4. doi: 10.1016/j.ajo.2005.08.018.

13. Vasavada V, Vasavada AR, Vasavada VA, Srivastava S, Gajjar DU, Mehta S. Incision integrity and postoperative outcomes after microcoaxial phacoemulsification performed using 2 incision-dependent systems. J Cataract Refract Surg. 2013;39(4):563-71. doi: 10.1016/j.jcrs.2012.11.018.

14. Berdahl JP, DeStafeno JJ, Kim T. Corneal wound architecture and integrity after phacoemulsification evaluation of coaxial, microincision coaxi$\mathrm{al}$, and microincision bimanual techniques. J Cataract Refract Surg. 2007;33(3):510-5. doi: 10.1016/j. jcrs.2006.11.012.

15. Calladine D, Ward M, Packard R. Adherent ocular bandage for clear corneal incisions used in cataract surgery. J Cataract Refract Surg. 2010;36(11):183948. doi: 10.1016/j.jcrs.2010.06.058.

16. McGowan BL. Mechanism for development of endophthalmitis. J Cataract Refract Surg. 1994;20(1):111.

17. Can I, Bayhan HA, Celik H, Bostancı Ceran B. Anterior segment optical coherence tomography evaluation and comparison of main clear corneal incisions in microcoaxial and biaxial cataract surgery. J Cataract Refract Surg. 2011;37(3):490-500. doi: 10.1016/j.jcrs.2010.09.024.
18. Xia Y, Liu X, Luo L, Zeng Y, Cai X, Zeng M, et al. Early changes in clear cornea incision after phacoemulsification: an anterior segment optical coherence tomography study. Acta Ophthalmol. 2009;87(7):764-8. doi: 10.1111/j.1755-3768.2008.01333.x

19. Calladine D, Packard R. Clear corneal incision architecture in the immediate postoperative period evaluated using optical coherence tomography. J Cataract Refract Surg. 2007;33(8):1429-35. doi: 10.1016/j.jcrs.2007.04.011.

20. Wang L, Dixit L, Weikert MP, Jenkins RB, Koch DD. Healing changes in clear corneal cataract incisions evaluated using Fourier-domain optical coherence tomography. J Cataract Refract Surg. 2012;38(4):660-5. doi: 10.1016/j.jcrs.2011.10.030.

21. Grewal DS, Basti S. Comparison of morphologic features of clear corneal incisions created with a femtosecond laser or a keratome. J Cataract Refract Surg. 2014;40(4):521-30. doi: 10.1016/j. jcrs.2013.11.028.

22. Teixeira A, Salaroli C, Filho FR, Pinto FT, Souza N, Sousa BA, et al. Architectural analysis of clear corneal incision techniques in cataract surgery using Fourier-domain OCT. Ophthalmic Surg Lasers Imaging. 2012;43(6 Suppl):S103-8. doi: 10.3928/15428877-20121003-02.

23. Mastropasqua L, Toto L, Mastropasqua A, Vecchiarino L, Mastropasqua R, Pedrotti E, et al. Femtosecond laser versus manual clear corneal incision in cataract surgery. J Refract Surg. 2014;30(1):27-33.

24. Ding $X$, Chang P, Savini G, Huang J, Wang Q, Lin $\mathrm{H}$, et al. Clear corneal incision architecture imaging after torsional phacoemulsification of hard nuclear cataract through a $2.2 \mathrm{~mm}$ and a $3.0 \mathrm{~mm}$ large tunnel. J Clin Exp Ophthalmol. 2013;4(3):1-6.

25. Alió JL, Mulet ME, Shalaby AMM, Attia WH. Phacoemulsification in the anterior chamber. J Cataract Refract Surg. 2002;28(1):67-75.

26. Holzer MP, Tetz MR, Auffarth GU, Welt R, Völcker HE. Effect of Healon 5 and 4 other viscoelastic substances on intraocular pressure and endothelium after cataract surgery. J Cataract Refract Surg. 2001;27(2):213-8.

27. Fang JP, Amesur KB, Baratz KH. Preexisting endothelial abnormalities in bilateral postoperative Descemet membrane detachment. Arch Ophthalmol. 2003;121(6):903-4. doi: 10.1001/archopht.121.6.903. 
28. Sharma N, Singhal D, Nair SP, Sahay P, Sreeshankar SS, Maharana PK. Corneal edema after phacoemulsification. Indian J Ophthalmol. 2017;65(12):1381-9. doi: 10.4103/ijo.IJO_871_17.

29. Fukuda S, Kawana K, Yasuno Y, Oshika T. Wound architecture of clear corneal incision with or without stromal hydration observed with 3-dimensional optical coherence tomography. Am J Ophthalmol. 2011;151(3):413-419.el. doi: 10.1016/j. ajo.2010.09.010.

30. McDonnell PJ, Taban M, Sarayba M, Rao B, Zhang J, Schiffman R, et al. Dynamic morphology of clear corneal cataract incisions. Ophthalmology. 2003;110(12):2342-8. doi: 10.1016/ S0161-6420(03)00733-4.

31. Shingleton BJ, Wadhwani RA, O'Donoghue MW, Baylus S, Hoey H. Evaluation of intraocular pressure in the immediate period after phacoemulsification. J Cataract Refract Surg. 2001;27(4):524-7.

32. Fine IH, Hoffman RS, Packer M. Profile of clear corneal cataract incisions demonstrated by ocular coherence tomography. J Cataract Refract Surg. 2007;33(1):94-7. doi: 10.1016/j.jcrs.2006.09.016.
33. Calladine D, Tanner V. Optical coherence tomography of the effects of stromal hydration on clear corneal incision architecture. J Cataract Refract Surg. 2009;35(8):1367-71. doi: 10.1016/j.jcrs.2009.03.036.

34. Lyles GW, Cohen KL, Lam D. OCT-documented incision features and natural history of clear corneal incisions used for bimanual microincision cataract surgery. Cornea. 2011;30(6):681-6. doi: 10.1097/ICO.0b013e31820128bb.

35. Calladine D, Packard R. Clear corneal incision architecture in the immediate postoperative period evaluated using optical coherence tomography. J Cataract Refract Surg. 2007;33(8):1429-35. doi: 10.1016/j.jcrs.2007.04.011.

36. Behrens A, Stark WJ, Pratzer KA, McDonnell PJ. Dynamics of small-incision clear cornea wounds after phacoemulsification surgery using optical coherence tomography in the early postoperative period. J Refract Surg. 2008;24(1):46-9. doi: 10.3928/1081597X-20080101-07. 